\title{
ПРОБЛЕМА ИСПОЛЬЗОВАНИЯ ОТХОДОВ ЛАКОКРАСОЧНЫХ МАТЕРИАЛОВ И ИХ УТИЛИЗАЦИЯ
}

\author{
Барханаджян Аида Левоновна 1 , \\ bal-37@mail.ru
}

\author{
Хакимов Равшан Муминович1, \\ hakimov-ravshan@mail.ru
}

Ибрагимов Ботир Дастамович1, ibragimov.bd@mail.ru

\section{Собирова Дилорам Кабуловна ${ }^{1}$}

\section{Абдукаримова Гульсуной Уринбаевна ${ }^{1}$}

\author{
Айрапетов Дмитрий Алексеевич1, \\ ayrapetov92@mail.ru \\ 1 Ташкентский институт по проектированию, строительству и эксплуатации автомобильных дорог, \\ Узбекистан, 100060, г. Ташкент, пр. А. Тимура, 20.
}

\begin{abstract}
Актуальность исследования. Коррозия металлов является наиважнейшей проблемой, с которой связаны большие потери металлов. Ежегодно от коррозии безвозвратно теряются сотни тонн металлов, поэтому все исследования, направленные на антикоррозионную защиту металлов, являются целесообразными и актуальными.

Цель исследования. Существуют различные методы антикоррозионной защиты металлических деталей, но нет достаточно эфффективного метода для конкретных условий антикоррозионной защиты. Целью настоящей работы является использование отходов лакокрасочных материалов для получения антикоррозионных покрытий металлических деталей.

Объектом исследования являются отходы лакокрасочных материалов, полученные при окраске автомобилей.

Методы: изучение физико-химических свойств отходов; подбор состава для растворения отходов лакокрасочных материалов; разработка рецептуры и получение антикоррозионной мастики; испытание полученного антикоррозионного лакокрасочного покрытия на адгезию, химостойкость, прочность пленки на изгиб, прочность пленки на удар, огнестойкость, морозостойкость; определение наиболее эфффективного компонентного и количественного состава полученного покрытия. Выбраны металлические пластинки для нанесения защитного лакокрасочного покрытия, растворы различных соединений для проведения испытаний по определению потери массы опытных металлических образцов

Результаты. Разработана и получена мастика на основе госсиполовой смолы и твердых отходов лакокрасочных материалов. Отходы лакокрасочных материалов - потери, получаемые при окраске автомобилей. Госсиполовая смола представляет собой отход масложиркомбината. Полученная мастика прошла испытания в лабораторных условиях по показателям качества в соответствии со стандартами, получены положительные результаты. Проведены эксплуатационные испытания в дорожных условиях, пробег автомобиля составил 12780 км, при исследовании изменения на поверхности покрытия не обнаружены.
\end{abstract}

\section{Ключевые слова:}

Отходы, лакокрасочные материалы, подготовка мастики, ингредиенты мастики, антикоррозионные свойства, адгезия, прочностные свойства, эксплуатационные испытания, защита металлов, эфффективность мастики.

\section{Введение}

Высокие темпы развития промышленных предприятий приводят к образованию большого количества отходов. В связи с этим в настоящее время проблема утилизации отходов является наиважнейшей задачей и решение ее может иметь, несомненно, и экономический, и экологический эффект [1].

Проблема использования отходов может быть решена созданием системы утилизации, т. е. возможности использования отходов после их вторичной переработки. Наиболее эффективным направлением утилизации этих отходов является использование их в качестве техногенного сырья при получении полезных технических продуктов.

В настоящее время общее годовое образование твердых отходов в мире составляет приблизительно
17 млрд т и к 2050 г. достигнет 27 млрд т [2]. Из этого количества около 1,3 млрд т в данное время составляют твердые бытовые отходы, образующиеся в городах мира [3]. Известно, что любые отходы создают неблагоприятную экологическую обстановку, такую как изменение климата, истощение стратосферного озона, ущерб здоровью человека, ущерб экосистеме и истощение ресурсов, и это лишь некоторые из них. Образование большого количества твердых отходов требует комплексно решать эти экологические проблемы, что может привести к более устойчивой экологической обстановке [4].

Учеными различных стран [5-8] проводятся многочисленные исследования, посвященные проблемам утилизации отходов. Ими предлагаются различные варианты переработки промышленных и бытовых отходов. Это, например, изменение технологического 
процесса, замена токсичных ингредиентов на безопасные и т. д. Так, на заводах по производству красок на основе растворителей образуются значительные количества опасных отходов, которые должны быть надлежащим образом обработаны, прежде чем их можно будет утилизировать. Поскольку стоимость обработки этих отходов высока, сокращение количества отходов стало решающей проблемой в этой отрасли.

Варианты минимизации отходов выгодны как для владельца завода, так и для окружающей среды. Количество опасных отходов можно свести к минимуму, изменив процесс производства или заменив вредные вещества, которые используются в процессе производства краски, на экологически чистые. Кроме того, разделение различных потоков отходов облегчает извлечение сырья и повышает вероятность его повторного использования в процессе производства. Это снизит эксплуатационные расходы завода [9].

Обстановка по образованию, размещению, накоплению, использованию и обезвреживанию отходов в Республике Узбекистан оценивается постепенным ростом количества образования и промышленных и бытовых отходов.

По статистическим данным в Республике Узбекистан в 2017 г. насчитывалось порядка 114,7 млн т отходов, из них токсичных отходов, подлежащих обязательной переработке, $-42,8$ млн т. Поэтому в стране проводится последовательная работа в области охраны окружающей среды, улучшения санитарной и экологической обстановки [10].

В целях охраны окружающей среды в настоящее время в Узбекистане насчитывается 296 мест захоронений и утилизации всех видов отходов.

Здесь следует отметить, что более интенсивно промышленные отходы образуются на предприятиях горно-нефтедобывающей, угольной и химической промышленностей. Такое положение объясняется отсутствием технологий по уменьшению их образования и повторного использования.

Вместе с тем в Республике Узбекистан ведутся работы по переработке отходов для получения вторичного сырья, в частности, это отходы газохимического комбината, шинного завода, лакокрасочных производств и др.

В связи с проблемой утилизации отходов в качестве возможного перспективного сырья для получения новой продукции нами рассмотрены отходы лакокрасочных материалов (ЛКМ).

Известно, что отходы лаков и красок по опасности относятся к 3-му классу токсичности, т. к. они содержат помимо растворителей, пластификаторов, наполнителей, пигментов и другие ингредиенты опасного характера, токсично влияющие на здоровье человека. К ним относятся тяжелые металлы, такие как свинец, фосфор, хром и кадмий [11-14].

Учитывая токсичность лакокрасочных материалов в ряде стран Европы, США, Японии были приняты нормы, ограничивающие содержание тяжелых металлов - свинца, фосфора и кадмия в красках $[15,16]$.
Известно, что до настоящего времени вопрос переработки отходов ЛКМ еще недостаточно изучен и имеющиеся материалы в отечественной и зарубежной литературе не дают достаточной информации по их применению. Хотя имеются некоторые технологии, применяемые для утилизации отходов ЛКМ, такие как у компании «General Motors», предусматривающие пиролиз, и технология компании «Haden», заключающаяся в обезвоживании отходов, его сушку с последующим измельчением [17].

Для решения заданной проблемы нами рассмотрена наиболее перспективная технология использования отходов ЛКМ в получении антикоррозионного покрытия. При этом были использованы отходы лакокрасочных материалов после окраски автомобиля на заводе CП OOO «Sam Auto» для получения антикоррозионной лакокрасочной мастики.

Анализ химического состава отходов показал наличие в нем следующих химических соединений: $\mathrm{Al}_{2} \mathrm{O}_{3}-1,0 \%, \mathrm{ZnO}-1,6 \%, \mathrm{SiO}_{2}-5,7 \%, \mathrm{TiO}_{2}-$ $5,65 \%, \mathrm{Fe}_{2} \mathrm{O}_{3}-0,19 \%, \mathrm{FeO}-1,44 \%, \mathrm{MgO}-7,4 \%$, $\mathrm{MnO}-0,07 \%, \mathrm{CaO}-15,7 \%, \mathrm{Na}_{2} \mathrm{O}-0,14 \%, \mathrm{BaO}-$ $0,17 \%, \mathrm{~K}_{2} \mathrm{O}-0,05 \%, \mathrm{P}_{2} \mathrm{O}_{5}-11,54 \%, \mathrm{H}_{2} \mathrm{O}-4,8 \%$, $\mathrm{CO}_{2}-4,95 \%, \mathrm{SO}_{3}-0,85 \%, \mathrm{~S}-0,7 \%$.

Как видно из вышеприведенного анализа, наиболее токсичным является оксид фосфора, который необходимо было перевести в нетоксичную форму.

Были отобраны отходы ЛКМ, подобран наиболее эффективный растворитель № 646 (смесь толуол $50 \%$, этанол - $15 \%$, бутилацетат (или амилацетат) $10 \%$, бутанол $-10 \%$, ацетон - $7 \%$ ) и др.), куда вводили измельченные до мелкодисперсного состояния отходы. После полного растворения отходов и образования однородной массы ее смешивали с предварительно подготовленной госсиполовой смолой, затем для быстрого высыхания вводили отвердитель для получения мастики. Госсиполовая смола образуется при обработке жиров и масел растворами щелочей и обладает активными функциональными группами [18]. При составлении мастики госсиполовую смолу предварительно обезвоживали.

Анализ на токсичность $\mathrm{P}_{2} \mathrm{O}_{5}$ полученного нами защитного покрытия приводится в табл. 1 [19].

Таблица 1. Содержание фосфора в образиах

Table 1. Phosphorus content in samples

\begin{tabular}{|l|c|}
\hline \multicolumn{1}{|c|}{$\begin{array}{c}\text { Образец } \\
\text { Sample }\end{array}$} & $\begin{array}{c}\text { Фосфор } \\
\text { Phosphorus }\left(\mathrm{P}_{2} \mathrm{O}_{5}\right), \\
\mathrm{mg} / \mathrm{kg}\end{array}$ \\
\hline отход ЛКМ/paint work waste & 91,0 \\
\hline $\begin{array}{l}\text { полученный продукт } \\
\text { received product }\end{array}$ & 7,6 \\
\hline ПДК/TLV & 27,2 \\
\hline
\end{tabular}

Как показали результаты анализов по токсичности, полученная антикоррозионная мастика существенно отличается от предельно допустимой концентрации (ПДК) по $\mathrm{P}_{2} \mathrm{O}_{5}$ и составляет 7,6 мг/кг.

Экспериментально был установлен наиболее эффективный компонентный состав антикоррозионной мастики (табл. 2). 
Таблица 2. Компонентный состав опытной антикоррозионной мастики

Table 2. Composition of the experimental anti-corrosion mastic

\begin{tabular}{|l|c|c|c|c|c|}
\hline \multirow{2}{*}{$\begin{array}{l}\text { Компонентный состав } \\
\text { Component composition }\end{array}$} & \multicolumn{5}{|c|}{ Опытные образцы мастик } \\
\cline { 2 - 6 } & № 1 & № 2 & № 3 & № 4 & № 5 \\
\hline $\begin{array}{l}\text { Госсиполовая смола } \\
\text { Gossypol resin, \% }\end{array}$ & 40 & 30 & 60 & 50 & 50 \\
\hline $\begin{array}{l}\text { Oтходы лакокрасочных } \\
\text { материалов }\end{array}$ & 40 & 50 & 15 & 20 & 30 \\
$\begin{array}{l}\text { Waste paint work } \\
\text { materials, \% }\end{array}$ & 15 & 15 & 20 & 20 & 15 \\
\hline $\begin{array}{l}\text { Pacrворитель } \\
\text { Thesolvent, \% }\end{array}$ & 5 & 5 & 5 & 10 & 5 \\
\hline $\begin{array}{l}\text { Oтвердитель } \\
\text { Hardener, \% }\end{array}$ & \multicolumn{7}{|c|}{} \\
\hline
\end{tabular}

Из таблицы видно, что полученная мастика состоит в основном из госсиполовой смолы $(30 \ldots 60 \%)$ и отходов лакокрасочных материалов (15.. 50 \%).

Для выявления наиболее подходящей композиции полученной мастики ее наносили на металлические пластинки и после высыхания определяли основные показатели качества полученных покрытий. Эксперименты проводили по каждому образцу в одинаковых условиях (табл. 3) [20].

Как следует из табл. 3 опытные образцы мастики 4 и 5 по качеству не уступают промышленному образцу марки «Барьер», а по морозостойкости превосходят. При повышении температуры на поверхности промышленного образца наблюдались трещины, в то время как лабораторные образцы остались без изменения, поэтому для последующих исследований нами были отобраны образцы 4 и 5.

Определение антикоррозионных свойств полученного покрытия проводили в различных агрессивных растворах. Подготовленные по ГОСТу 9.080-77 пла- стины из стали, меди, алюминия, латуни, чугуна с покрытием погружали в растворы $\mathrm{H}_{2} \mathrm{O}, \mathrm{NaCl}, \mathrm{Na}_{2} \mathrm{CO}_{3}$, $\mathrm{CaCO}_{3}$, с концентрацией $0,1 \ldots 0,15 \%$ и выдерживали в течении 12 часов при температуре $40{ }^{\circ} \mathrm{C}$. Растворы солей были выбраны в соответствии с условиями эксплуатации автомобилей и с учетом засоленности почвы в ряде областей республики (табл. 4).

Из таблицы видно, что потери массы испытуемых пластин несущественны и составляют от 0,001 до 0,004 г. Визуально поверхность пластин не потеряла блеска и не наблюдались изменения в виде шероховатостей и трещин.

Для эксплуатационных испытаний готовили пластинки с нанесением опытной мастики и промышленного образца марки «Барьер». Пластинки с такими покрытиями прикреплялись на автомобиль в местах, наиболее подверженных загрязнению, коррозии и гравитационному повреждению. Результаты эксплуатационных испытаний приведены в табл. 5.

После пробега автомобиля 12,780 тыс. км пластинки были сняты и проанализированы. Визуальный анализ показал, что лабораторный образец практически остался без изменения, а образец с промышленным покрытием был сильно поврежден, при этом потери массы составили для лабораторного образца 0,12 г, а для промышленного - 0,18 г. Следует отметить, что эксплуатационные испытания проводились в тяжелых погодных условиях при высокой влажности, в морозную и дождливую погоду в городских и загородных условиях.

Из результатов экспериментальных испытаний следует, что полученные покрытия на основе отходов ЛКМ и госсиполовой смолы по показателям качества соответствуют требованиям, предъявляемым к защитным покрытиям.

Таблица 3. Испытания полученных опытных образиов в лабораторных условиях

Table 3. Laboratory tests of the obtained prototypes

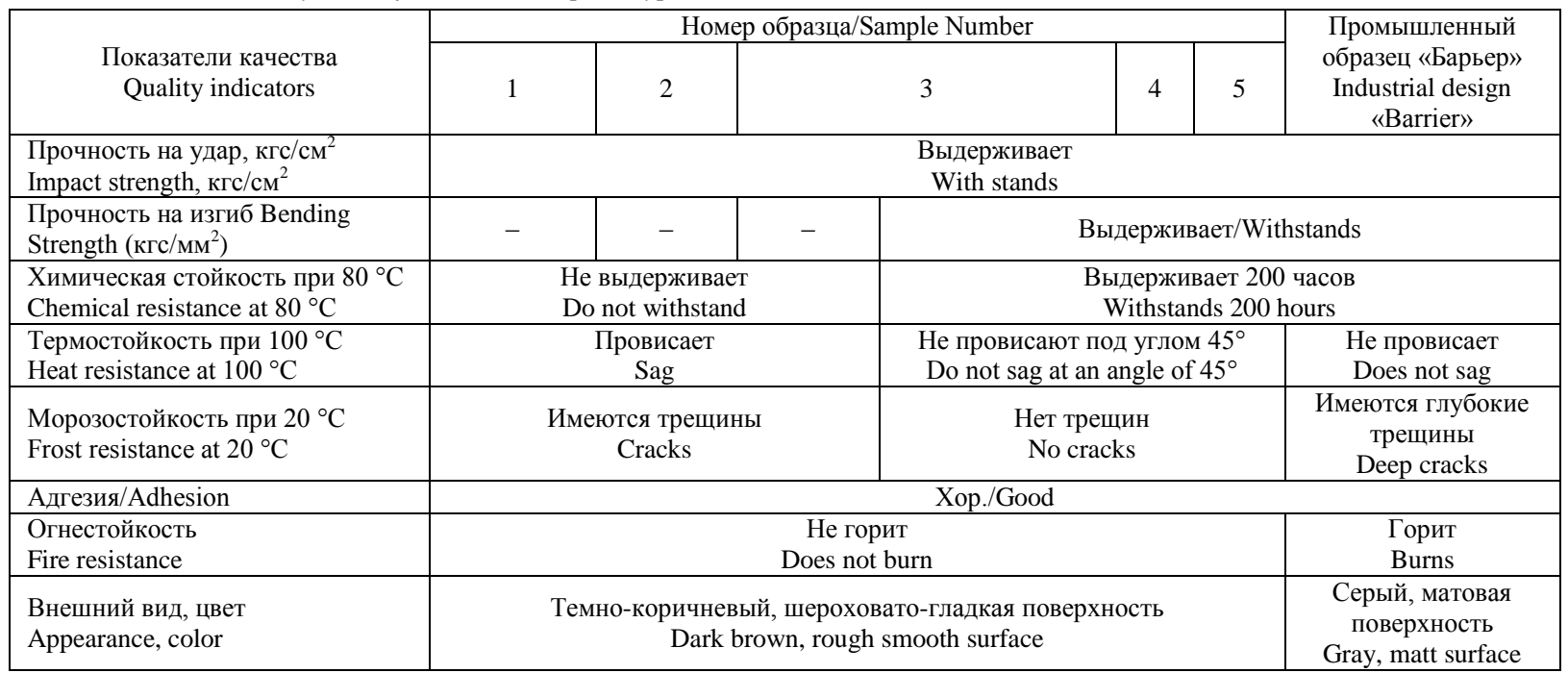


Таблица 4. Коррозия металлов в растворах различных сред при конщентрациях $0,1 \ldots 0,15 \%$

Table 4. Corrosion of metals in solutions of various media at concentrations $0,1 \ldots 0,15 \%$

\begin{tabular}{|c|c|c|c|c|c|c|c|}
\hline $\begin{array}{c}\text { Растворы раз- } \\
\text { личных сред } \\
\text { Solutions of } \\
\text { different media }\end{array}$ & & Образец/Sample & Сталь/Steel & Медь/Copper & $\begin{array}{l}\text { Алюминий } \\
\text { Aluminum }\end{array}$ & $\begin{array}{c}\text { Латунь } \\
\text { Brass Cast }\end{array}$ & $\begin{array}{l}\text { Чугун } \\
\text { Iron }\end{array}$ \\
\hline \multirow{3}{*}{ 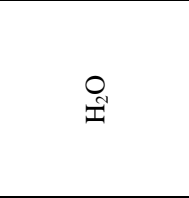 } & \multirow{12}{*}{ 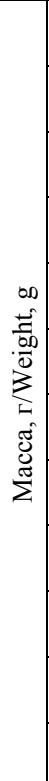 } & $\begin{array}{l}\text { До опыта } \\
\text { Before the experiment }\end{array}$ & 12,713 & 4,812 & 2,774 & 6,632 & 6,818 \\
\hline & & $\begin{array}{l}\text { После опыта } \\
\text { After the experiment }\end{array}$ & 12,713 & 4,812 & 2,774 & 6,632 & 6,817 \\
\hline & & $\begin{array}{l}\text { Потеря массы } \\
\text { Mass loss }\end{array}$ & 0 & 0 & 0 & 0 & 0,001 \\
\hline \multirow{3}{*}{$\overrightarrow{\widetilde{Z}}$} & & $\begin{array}{l}\text { До опыта } \\
\text { Before the experiment }\end{array}$ & 12,713 & 4,815 & 2,805 & 6,630 & 6,851 \\
\hline & & $\begin{array}{l}\text { После опыта } \\
\text { After the experiment }\end{array}$ & 12,711 & 4,811 & 2,805 & 6,628 & 6,849 \\
\hline & & $\begin{array}{l}\text { Потеря массы } \\
\text { Mass loss }\end{array}$ & 0,002 & 0,004 & 0 & 0,002 & 0,002 \\
\hline \multirow{3}{*}{ 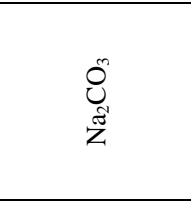 } & & $\begin{array}{l}\text { До опыта } \\
\text { Before the experiment }\end{array}$ & 12,7 & 4,805 & 2,695 & 6,645 & 6,853 \\
\hline & & $\begin{array}{l}\text { После опыта } \\
\text { After the experiment }\end{array}$ & 12,699 & 4,801 & 2,695 & 6,644 & 6,851 \\
\hline & & $\begin{array}{l}\text { Потеря массы } \\
\text { Mass loss }\end{array}$ & 0,001 & 0,004 & 0 & 0,001 & 0,002 \\
\hline \multirow{3}{*}{ 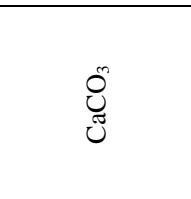 } & & $\begin{array}{l}\text { До опыта } \\
\text { Before the experiment }\end{array}$ & 12,785 & 4,816 & 2,711 & 6,651 & 6,905 \\
\hline & & $\begin{array}{l}\text { После опыта } \\
\text { After the experiment }\end{array}$ & 12,783 & 4,814 & 2,711 & 6,650 & 6,902 \\
\hline & & $\begin{array}{l}\text { Потеря массы } \\
\text { Mass loss }\end{array}$ & 0,002 & 0,002 & 0 & 0,001 & 0,003 \\
\hline
\end{tabular}

Таблица 5. Результаты эксплуатационных испытаний

Table 5. Results after testing

\begin{tabular}{|c|c|c|c|c|}
\hline \multirow[t]{2}{*}{ Показатели/Indicators } & \multicolumn{2}{|c|}{ Опытный образец/Prototype } & \multicolumn{2}{|c|}{$\begin{array}{c}\text { Промышленный образец марки «Барьер» } \\
\text { Industrial model «Barrier» } \\
\end{array}$} \\
\hline & $\mathrm{I}_{1}$ & $\mathrm{II}_{1}$ & $\mathrm{I}_{2}$ & $\mathrm{II}_{2}$ \\
\hline $\begin{array}{l}\text { Размер пластинок, см } \\
\text { size of the plates, } \mathrm{cm}\end{array}$ & \multicolumn{4}{|c|}{$10 * 10$} \\
\hline $\begin{array}{l}\text { Macca пластинки до опыта без мастики, г } \\
\text { Plate weight before the experiment without mastic, g }\end{array}$ & 100,3 & 98,75 & 98,7 & 96,95 \\
\hline $\begin{array}{l}\text { Macca пластинки с мастикой до опыта, г } \\
\text { Weight of plate with mastic before the experiment, g }\end{array}$ & 105,37 & 99,72 & 103,85 & 97,90 \\
\hline $\begin{array}{l}\text { Macca пластинки с мастикой после опыта, г } \\
\text { Weight of the plate with mastic after the experiment, g }\end{array}$ & 105,22 & 99,60 & 103,65 & 97,72 \\
\hline $\begin{array}{l}\text { Пробег автомобиля, тыс. км } \\
\text { Vehicle mileage, thousand km }\end{array}$ & 12,780 & 12,780 & 12,780 & 12,780 \\
\hline
\end{tabular}

Особенностью данной работы является использование отходов для получения нужных технических продуктов, что важно, как с экологической, так и с экономической точки зрения.

По известным данным на окраску одного автомобиля расходуется порядка 30-35 кг краски. Потери при окраске составляют 50 \% в виде отходов. Учитывая, что ежегодно завод СП $\mathrm{OOO}$ «Sam Auto» выпускает в среднем 4000 различных моделей автомобилей, эти потери составят более 35-50 т, которые не перерабатываются, не утилизируются, а закапываются в могильнике, при этом заражается огромная площадь земли, для восстановления которой потребуются огромные затраты.

Таким образом, возможность использования лакокрасочных отходов только на одном заводе существенно улучшит экологическую обстановку, исключается необходимость в могильниках и заражение почвы.

\section{Заключение}

1. Получено новое антикоррозионное покрытие на основе отходов ЛКМ и госсиполовой смолы.

2. Результаты испытаний антикоррозионного покрытия показали, что по прочности на удар, на изгиб, химостойкости, морозостойкости, огнестойкости, адгезии оно отвечает требованиям, предъявляемым к антикоррозионным покрытиям, и является не токсичным.

3. Полученное антикоррозионное покрытие может быть рекомендовано для покрытия днища, внутренней поверхности крыльев, кузова автомобилей, деталей сельскохозяйственной техники, а также может быть использовано в автосервисных и других производствах.

4. Применение отходов для получения антикоррозионной мастики в автомобильной промышленности и дорожно-строительной технике, несомненно, даст как экологический, так и экономический эффект. 


\section{СПИСОК ЛИТЕРАТУРЬ}

1. Заболотских В.В., Васильев А.В., Батова К.Э. Анализ проблемы образования отходов лакокрасочных материалов в самарской области и перспективы их переработки // Известия Самарского научного центра Российской академии наук. 2015. - T. 17. - № 4. - C. 273-276.

2. Karak T., Bhagat R.M., Bhattacharyya P. Municipal solid waste generation, composition, and management: the world scenario // Critical Reviews in Environmental Science and Technology. 2012. - V. 42. - № 15. - P. 1509-1630. URL: https://doi.org/ 10.1080/10643389.2011.569871 (дата обращения 28.08.2020).

3. Hoornweg D., Bhada-Tata P. What a waste - a global review of solid waste management // World Bank. - 2012. - № 15. - P. 116 URL: https://openknowledge.worldbank.org/handle/10986/17388 (дата обращения 28.08.2020)

4. Review of LCA studies of solid waste management systems. P. I: Lessons learned and perspectives / A. Laurent, I. Bakas, J Clavreul, A. Bernstad, M. Niero, E. Gentil, M.Z. Hauschild, T.H. Christensen // Waste Management. - 2014. - V. 34. - № 3. P. 573-588. URL: https://doi.org/10.1016/j.wasman.2013.10.045

5. Vassilis J.I., Konstantinos M. Household hazardous waste management: a review // Journal of Environmental Management. 2015. - V. 150. - P. 310-321. URL: https://doi.org/10.1016/ j.jenvman.2014.11.021 (дата обращения 28.08.2020).

6. Mostafa M.K., Peters R.W. Reuse paint wastewater in the manufacture of cement bricks and tiles // Journal of Material Cycles and Waste Management. - 2017. - V. 19. - P. 840-850.

7. Eugenio F.D., Pajarito B.B. Potential anti-corrosion additives derived from waste plastic sachets // IOP Conference Series: Materials Science and Engineering. - 2019. - V. 634. - № 1. P. 1-6. URL: https://www.researchgate.net/publication/336531640 Potential_anti-corrosion_additives_derived_from_waste_plastic_ sachets (дата обращения 28.08.2020).

8. Mohamed M.G., Ahmed N.M., Abd El-Gawad W.M. Corrosion protection performance of reinforced steel coated with paints based on waste materials // Anti-Corrosion Methods and Materials. - 2018. - V. 65. - № 4. - P. 368-374.

9. Dursun D., Sengul F. Waste minimization study in a solvent-based paint manufacturing plant // Resources, conservation and recycling. - 2006. - V. 47. - № 4. - P. 316-331.

10. Указ Президента Республики Узбекистан № УП-5863 от 30.10.2019 «Об утверждении концепции охраны окружающей среды Республики Узбекистан до 2030 года». - Ташкент, 2019. -43 с

11. Козыренко М.И., Кухарчик Т.И. Загрязнение почв при производстве лакокрасочных материалов // Геоэкология. Инженерная геология, гидрогеология, геокриология. - 2015. - № 3. C. 230-238.

12. Obeng-Gyasi E. Sources of lead exposure in various countries // Reviews on environmental health. - 2019. - V. 34. - № 1. - P. 25-34.

13. Characterisation of silicon, zirconium and aluminium coated titanium dioxide pigments recovered from paint waste / M.C. Karlsson, Z. Abbas, R. Bordes, Y. Cao, A. Larsson, P. Taylor, B. Steenari // Dyes and pigments. - 2019. - V. 162. P. $145-152$.

14. A simple and effective process for recycling zinc-rich paint residue / P. Xing, B. Ma, C. Wang, L. Wang, Y. Chen // Waste management. - 2018. - V. 76. - P. 234-241.

15. Developing common core criteria for paints. Final Report submitted to Global Eco-labeling Network. - Bangkok: Thailand Environment Institute, 2003. - 17 p. URL: https:// globalecolabelling.net/assets/Uploads/tech-030522-02.pdf (дата обращения 28.08.2020).

16. Double standard. Investigating lead $(\mathrm{Pb})$ content in leading enamel paint brands in South Asia. - New Delhi: Toxics Link, 2011. 36 p. URL: http://toxicslink.org/docs/Double_Standard_Lead_ Paint 29 June 2011.pdf (дата обращения 28.08.2020).

17. Доусон $\bar{\Gamma}$., Мерсер Б. Обезвреживание токсичных отходов / Пер. с англ. - М.: Стройиздат, 1996. - 288 с.

18. The inhibition of the corrosion of carbon steel by oligomeric corrosion inhibitors in different media / K.S. Beknazarov, A.T. Dzhalilov, U.Y. Ostanov, A.M. Erkaev // International Polymer Science and Technology. - 2015. - V. 42. - № 4. P. 33-37.

19. Использование отходов лакокрасочных материалов для получения полезных технических продуктов / А.Л. Барханаджян, Р.М. Хакимов, Р. Азизов, Т. Валитов // Актуальные проблемы автомобильно-дорожного комплекса Узбекистана: Сборник материалов республиканской научно-практической конференции. - Ташкент, 2010. - Ч. 1. - С. 67-69.

20. Проблема утилизации отходов лакокрасочных материалов / Р.М. Хакимов, Т.А. Садыков, Р. Азизов, Т. Валитов // Вестник ТАДИ Ташкент. - 2015. - № 1. - С. 103-106.

Поступила 04.09.2020 2.

\section{Информация об авторах}

Барханаджян А.Л., кандидат химических наук, доцент кафедры экологии и двигателей внутреннего сгорания Ташкентского института по проектированию, строительству и эксплуатации автомобильных дорог.

Хакимов P.M., кандидат технических наук, доцент кафедры экологии и двигателей внутреннего сгорания Ташкентского института по проектированию, строительству и эксплуатации автомобильных дорог.

Ибрагимов Б.Д., старший преподаватель кафедры эксплуатации автомобильного транспорта Ташкентского института по проектированию, строительству и эксплуатации автомобильных дорог.

Собирова Д.К., кандидат технических наук, старший преподаватель кафедры естественных наук Ташкентского института по проектированию, строительству и эксплуатации автомобильных дорог.

Абдукаримова $Г$.У., старший преподаватель кафедры эксплуатации автомобильного транспорта Ташкентского института по проектированию, строительству и эксплуатации автомобильных дорог.

Aйрапетов Д.А., ассистент кафедры экологии и двигателей внутреннего сгорания Ташкентского института по проектированию, строительству и эксплуатации автомобильных дорог. 
UDC 628.477.6:620.193.4

\title{
PROBLEM OF USING WASTE OF PAINT AND VARNISHING MATERIALS AND THEIR DISPOSAL
}

\author{
Aida L. Barkhanadzhyan 1 , \\ bal-37@mail.ru
}

Ravshan M. Khakimov'1, hakimov-ravshan@mail.ru

Botir D. Ibragimov', ibragimov.bd@mail.ru

\section{Diloram K. Sobirova ${ }^{1}$}

\section{Gulsunoy U. Abdukarimova1}

Dmitriy A. Ayrapetov', ayrapetov92@mail.ru

1 Tashkent Institute of Design, Construction \& Maintenance of Automotive Roads, 20, A. Temur avenue, Tashkent, 100060, Uzbekistan.

The relevance of the research. Corrosion of metals is the most important problem with which large losses of metals are associated. Hundreds of tons of metals are irretrievably lost annually from corrosion, therefore, all studies aimed at the corrosion protection of metals are appropriate and relevant.

The main aim. There are various methods of anticorrosion protection of metal parts, but there is no sufficiently effective method for specific conditions of anticorrosion protection. The aim of this work is the use of waste paint and varnish materials to obtain anti-corrosion coatings of metal parts.

Objects: waste paint and varnish materials obtained by painting cars.

Methods: study of the physicochemical properties of the waste; selection of compositions for dissolving paintwork materials; formulation development and receipt of anticorrosive mastic; testing the obtained anticorrosive paint coating for adhesion, chemical resistance, film strength in bending, impact strength, fire resistance, frost resistance; preparation of metal plates for applying a protective paint coating and solutions of various compounds for testing to determine the mass loss of experimental metal samples; determination of the most effective component and quantitative composition of the resulting coating.

Results. The authors have developed and obtained the mastic based on gossypol resin and solid waste of paints and varnishes. Gossypol resin is a waste oil-fat plant. The resulting mastic was tested in laboratory conditions in terms of quality, positive results were obtained. Operational tests were carried out in road conditions, the car's mileage was $12,780 \mathrm{~km}$, no changes were found on the surface of the coating.

\section{Key words:}

Waste, paints and varnishes, mastic preparation, mastic ingredients, anticorrosion properties, adhesion, strength properties, performance tests, metal protection, mastic efficiency.

\section{REFERENCES}

1. Zabolotskikh V.V., Vasiliev A.V., Batova K.E. Analysis of the problem of the formation of waste paint and varnish materials in the Samara region and the prospects for their processing. Bulletin of the Samara Scientific Center of the Russian Academy of Sciences, 2015, vol. 17, no. 4, pp. 273-276. In Rus.

2. Karak T., Bhagat R.M., Bhattacharyya P. Municipal solid waste generation, composition, and management: the world scenario. Critical Reviews in Environmental Science and Technology, 2012, vol. 42 , no. 15 , pp. 1509-1630. Available at: https://doi.org/ 10.1080/10643389.2011.569871 (accessed 28 August 2020).

3. Hoornweg D., Bhada-Tata P. What a waste - a global review of solid waste management. World Bank, 2012, no. 15, pp. 116 Available at: https://openknowledge.worldbank.org/handle/10986/ 17388 (accessed 28 August 2020).

4. Laurent A., Bakas I., Clavreul J., Bernstad A., Niero M., Gentil E., Hauschild M.Z., Christensen T.H. Review of LCA studies of solid waste management systems - Part I: Lessons learned and perspectives. Waste Management, 2014, vol. 34, no. 3, pp. 573588. Available at: https://doi.org/10.1016/j.wasman.2013.10.045 (accessed 28 August 2020).

5. Vassilis J.I., Konstantinos M. Household hazardous waste management: a review. Journal of Environmental Management, 2015, vol. 150, pp. 310-321. Available at: https://doi.org/10.1016/ j.jenvman.2014.11.021 (accessed 28 August 2020).
6. Mostafa M.K., Peters R.W. Reuse paint wastewater in the manufacture of cement bricks and tiles. Journal of Material Cycles and Waste Management, 2017, vol. 19, pp. 840-850.

7. Eugenio F.D., Pajarito B.B. Potential anti-corrosion additives derived from waste plastic sachets. IOP Conference Series: Materials Science and Engineering, 2019, vol. 634, no. 1, pp. 1-6. Available at: https://www.researchgate.net/publication/336531640_ Potential_anti-

corrosion_additives_derived_from_waste_plastic_sachets (accessed 28 August 2020).

8. Mohamed M.G., Ahmed N.M., Abd El-Gawad W.M. Corrosion protection performance of reinforced steel coated with paints based on waste materials. Anti-Corrosion Methods and Materials, 2018, vol. 65, no. 4, pp. 368-374.

9. Dursun D., Sengul F. Waste minimization study in a solvent-based paint manufacturing plant. Resources, conservation and recycling, 2006, vol. 47, no. 4, pp. 316-331.

10. Ukaz Prezidenta Respubliki Uzbekistan № UP-5863 ot 30.10.2019 «Ob utverzhdenii kontseptsii okhrany okruzhayushchei sredy Respubliki Uzbekistan do 2030 goda» [Decree of the President of the Republic of Uzbekistan No. UP-5863 dated 10/30/2019 «On approval of the concept of environmental protection of the Republic of Uzbekistan until 2030»]. Tashkent, 2019. 43 p.

11. Kozyrenko M.I., Kukharchik T.I. Soil pollution in the manufacture of paints and varnishes]. Geoecology. Engineering geology, hydrogeology, geocryology, 2015, no. 3, pp. 230-238. In Rus. 
12. Obeng-Gyasi E. Sources of lead exposure in various countries. Reviews on environmental health, 2019, vol. 34, no. 1, pp. 25-34.

13. Karlsson M.C., Abbas Z., Bordes R., Cao Y., Larsson A., Taylor P., Steenari B. Characterisation of silicon, zirconium and aluminium coated titanium dioxide pigments recovered from paint waste. Dyes and pigments, 2019, vol. 162, pp. 145-152.

14. Xing P., Ma B., Wang C., Wang L., Chen Y. A simple and effective process for recycling zinc-rich paint residue. Waste management, 2018, vol. 76, pp. 234-241.

15. Developing common core criteria for paints. Final Report submitted to Global Eco-labeling Network. Bangkok, Thailand Environment Institute, 2003. 17 p. Available at: https:// globalecolabelling.net/assets/Uploads/tech-030522-02.pdf (accessed 28 August 2020).

16. Double standard. Investigating lead $(\mathrm{Pb})$ content in leading enamel paint brands in South Asia. New Delhi, Toxics Link, 2011, p $36 . \quad$ Available at: http://toxicslink.org/docs/Double_Standard_Lead_ Paint_29_June_2011.pdf (accessed 28 August 2020).

17. Dawson G., Mercer B. Obezvrezhivanie toksichnykh otkhodov [Neutralization of toxic waste]. Translated from English. Moscow, Stroyizdat Publ., 1996. 288 p.

\section{Information about the authors}

Aida L. Barkhanadzhyan, Cand. Sc., associate professor, Tashkent Institute of Design, Construction \& Maintenance of Automotive Roads.

Ravshan M. Khakimov, Cand. Sc., associate professor, Tashkent Institute of Design, Construction \& Maintenance of Automotive Roads.

Botir D. Ibragimov, senior lecturer, Tashkent Institute of Design, Construction \& Maintenance of Automotive Roads. Diloram K. Sobirova, Cand. Sc., senior lecturer, Tashkent Institute of Design, Construction \& Maintenance of Automotive Roads.

Gulsunoy U. Abdukarimova, senior lecturer, Tashkent Institute of Design, Construction \& Maintenance of Automotive Roads.

Dmitriy A. Ayrapetov, assistant, Tashkent Institute of Design, Construction \& Maintenance of Automotive Roads. 